\title{
Diacronie
}

Studi di Storia Contemporanea

$N^{\circ} 16,4 \mid 2013$

Le monarchie nell'età dei nazionalismi

\section{Il patriottismo e la stampa ottomana}

\section{Luca Zuccolo}

\section{(2) OpenEdition \\ Journals}

\section{Edizione digitale}

URL: http://journals.openedition.org/diacronie/933

DOI: 10.4000/diacronie.933

ISSN: 2038-0925

\section{Editore}

Association culturelle Diacronie

\section{Notizia bibliografica digitale}

Luca Zuccolo, « II patriottismo e la stampa ottomana », Diacronie [Online], N 16, 4 | 2013, documento 10, Messo online il 01 décembre 2013, consultato il 01 mai 2019. URL : http://

journals.openedition.org/diacronie/933 ; DOI : 10.4000/diacronie.933 


\section{Diacronie}

10/

\section{Il patriottismo e la stampa ottomana}

Luca ZUCCOLO*

Attraverso lo studio della stampa quotidiana degli anni Ottanta del XIX secolo si intende analizzare la formazione delle idee patriottiche all'interno della società Ottomana e il ruolo chiave svolto dal Sultano e dalla sua corte come promotore $e$ ideatore dei simboli e della nuova identità ottomana "inventata" dalle élites con il fine di salvare e rilanciare l'Impero.

\section{La politica di Abdülhamid II tra 1880 e 1885, uno sguardo d'insieme}

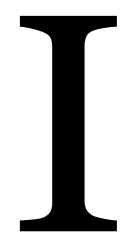

1 regno Hamidiano, la sua evoluzione e il suo dominatore, Abdülhamid II (18761908), sono stati a lungo obliati, sia dalla Repubblica di Turchia, la quale accusa il Sultano di essere stato un sovrano oscurantista e dispotico, anticostituzionale, nonché pan-islamista, sia dall'Europa, che biasima il regime poliziesco e censorio del Sultano rosso. Questa damnatio memoriae ha subito una svolta quando la storiografia ha iniziato a riscoprire la figura di Abdülhamid II $^{1}$, riconsiderando la vita e l'operato di un Sultano decisivo per gli sviluppi della società ottomano-turca, come sosteneva, già nel 1896, Paul Baudin, contrastando le visioni orientaliste $^{2}$ e il luogo comune di considerare il governo della Turchia come l'ideale del despotismo e dell'assolutismo:

${ }^{1}$ GEORGEON, François, Abdülhamid II, Le Sultan-Calife (1876-19o8), Paris, Fayard, 2003; KARPAT, Kemal H., The Politicization of Islam, Reconstructing Identity, State, Faith, and Community in the Late Ottoman State, New York, Oxford University press, 2001, pp. 158-164. ${ }^{2}$ ÇIRAKMAN, Asli, «From Tyranny to Despotism: The Enlightenment's Unenlightened Image of the Turks», in International Journal of Middle East Studies, 33, 1/2001, pp. 49-68. 
plus que toutes les constitutions imaginables, ce qui assure des droits qu'ont les citoyens en échange de leur devoirs envers l'Etat, c'est le respect de la loi de la part des gouvernants et l'obéissance à la loi de la part des gouvernés. Or ces respect cette obéissance ne se trouvent nulle part à un aussi haut degré qu'en Turquie3.

L'era Hamidiana, quindi, non è un periodo oscuro e di regressione, dominato dal fallimento del primo progetto costituzionale e parlamentare (1876-1878); al contrario, è l'apice delle riforme ottomane, trait d'union tra l'Impero e la Repubblica, poiché proprio durante questo trentennio l'Impero, seppur molto lentamente, si avvia verso la modernizzazione, senza che le precedenti strutture socio-politiche vengano abbandonate. Su questo regno, infatti, si protraggono le ombre dei Sultani precedenti, lo spirito riformista e modernizzatrice dei Giovani Ottomani e tutta la tradizione ottomano-islamica. Abdülhamid II non è altro che un «enfant des Tanzimat»; se non un attore principale, per lo meno un testimone privilegiato di questi anni di rinascita dell'Impero, e, allo stesso tempo, è un «enfant de la question d'orient» ${ }^{4}$. Inoltre, non si può dimenticare che il Sultano è anche un «Sultan bourgeoise», un monarca al passo con i tempi, che non vuole certo privarsi dei confort e delle comodità della vita moderna. Un imborghesimento che mostra a qual punto i modelli culturali occidentali siano penetrati nell'Impero5 come documenta il quotidiano «Stamboul» quando descrive i ricevimenti pubblici e privati del Sultano, tra cui spiccano quelli in onore di docenti e studenti delle nuove scuole civili imperiali ${ }^{6}$. Un esempio dell'adesione del Sultano alla modernità, e della sua benevolenza verso i sudditi espressione di un simbolismo inedito nella società ottomana.

Abdülhamid II, nei suoi trent'anni di regno, adotta una retorica e una politica ampiamente patriottiche, che consentono di inserire il discorso Hamidiano all'interno del panorama politico Europeo. Il fine del saggio pertanto è quello di identificare i vari strumenti materiali e ideologici utilizzati dal Sultano e dalle élites ottomane alla fine dell'Ottocento per costruire e immaginare l'Impero in chiave moderna. Si vuole, quindi, dimostrare come negli anni Ottanta dell'Ottocento si è sviluppata all'interno

3 BAUDIN, Paul, La Turquie et les Ottomans. Paris, s.e., 1896, pp. 116-117.

4 GEORGEON, François, Abdülhamid II, cit., p. 19.

5 GEORGEON, François, Abdülhamid II, cit., p. 141.

${ }^{6}$ Cfr. il trafiletto anonimo in cui si ricorda il dono fatto al Sultano da cinque studenti della scuola militare Stamboul, 14 febbraio 1881; il trafiletto «decorations», Stamboul, 7 gennaio 1881, relativo alle decorazioni donate ai docenti delle nuove scuole imperiali; e l'articolo «L'école de médecine a Yildiz» Stamboul, del 29 maggio 1882, in cui si descrive una visita degli studenti della scuola di medicina al palazzo e al parco di Yildiz. Un posto speciale ha il trafiletto anonimo in Stamboul 22 novembre 1883 sull'apertura, voluta dal Sultano, di una scuola femminile, simbolo della modernità dell'Impero, e nuovo strumento per legittimare i progetti Hamidiani di costruzione della nuova identità ottomana. 
dell'Impero Ottomano una dinamica socio-politico-culturale tale da porre alcune delle premesse del nazionalismo turco del XX secolo. Gli anni dal 1876 al 1909, di fatto, sono un periodo critico; l'Impero si dibatte all'interno di una «nationally imagined community», in cui l'identità ottomana assume sempre più marcati caratteri Turchi, sebbene si esprima nei termini dell'universalismo Islamico7 ${ }^{7}$. Il regno di Abdülhamid II si presenta come una realtà fluida progressivamente sclerotizzatasi su base protonazionale. Un processo che si sviluppa, a partire dagli anni 1880, in tutti i gruppi sociali imperiali ${ }^{8}$, sia tra le minoranze cristiane - Greci, Armeni o Levantini - sia attraverso gruppi dissidenti Ottomani come i Giovani Turchi.

Attraverso la stampa come mezzo di comunicazione e diffusione di idee e cultura e, in particolar modo, la stampa francofona come medium intercomunitario, esaminerò il sorgere e il primo evolversi di una formazione discorsiva di tipo patriottico che interessa tutte le comunità e i gruppi sociali e si pone prima in contrasto, poi in concorrenza, con la struttura sociopolitica tradizionale. Questo approccio permette non solo di analizzare e comprendere la fluidità della società ottomana ma, allo stesso tempo, consente di riformulare e riconsiderare la tempistica e la spazialità del nazionalismo postottomano. L'eredità ottomana e, in particolare l'eredità riformista ottocentesca e Hamidiana, sono fattori decisivi per la comprensione degli sviluppi nazionalistici turchi sia dell'epoca Kemalista sia di quella contemporanea.

Abdülhamid II, salito al trono nel 1876 e superata la crisi balcanica del 1877-78, infatti, sviluppa una politica peculiare. Un mix di riforme, tecnologia occidentale e recupero dei valori e delle tradizioni islamiche. I primi anni di regno (1876-1885) del Sultano rosso ${ }^{9}$ si presentano pertanto molto sfaccettati e articolati ${ }^{10}$. Durante questo periodo, si riuniscono tutti i fili del lungo passato ottomano e, al tempo stesso, si pongono le basi per i successivi sviluppi sociopolitici della Turchia contemporanea. Questo significa che dobbiamo chiederci come mai il regno Hamidiano ha avuto tanto successo ed è riuscito a preservare l’Impero Ottomano fino al 1918.

\footnotetext{
7 DERINGIL, Selim, The Well Protected Domains, Ideology and the Legitimation of Power in Ottoman Empire 1876-1909, London-New York, I.B. Tauris, 1998, p. 10.

${ }^{8}$ Una conferma della tesi di una progressiva sclerosi nazionale alla fine del XIX secolo si trova negli studi di Marie-Carmen Smyrnelis: SMYRNELIS, Marie-Carmen, Une Société hors de soi, identités et relations sociales à Smyrne aux XVIII e et XIX ${ }^{e}$ siècles, Paris, Peeters, 2005; ID., Une Ville ottoman plurielle, Smyrne aux XVIII ${ }^{e}$ et XIX ${ }^{e}$ siècle, Istanbul, ISIS, 2006.

9 Questo soprannome nasconde la dura accusa mossa contro Abdülhamid dagli orientalisti e dall'opinione pubblica Europea a causa dei massacri bulgari del 1876-1877 e del pogrom contro gli Armeni del 1894-95.

10 Per comprendere come gli Europei si rappresentavano e criticavano Abdülhamid II sono molto utili i saggi di Sir PEARS, Edwin, Life of Abdul Hamid, London, Constable \& Co. Ltd, Basil Williams, 1917.
} 
La risposta a questa domanda si trova da un lato, nei fallimenti delle Tanzimat (1839-76) ${ }^{11}$; dall'altro, nel progetto di uno Stato forte che riordini un Impero dominato da clan e signori regionali (ayan) ${ }^{12}$. Gli insuccessi delle riforme liberali, infatti, spingono molti membri delle élites a cercare delle alternative rintracciate nella politica e nell'ideologia Hamidiane le quali propongono un sistema in cui l'Islam ritorna centro dello sviluppo ideologico e permette di godere delle nuove scoperte tecnico-scientifiche senza contravvenire alla legge coranica ${ }^{13}$. La centralizzazione, invece, si inscrive nel processo di «modernizzazione difensiva» che interessa tutta l'area medio-orientale durante il XIX secolo e trova in Abdülhamid II un valido interprete ${ }^{14}$. Una riorganizzazione che permette di controllare meglio le periferie avvicinando le popolazioni allo Stato e creando un réseau sociopolitico in grado di diffondere le nuove idee provenienti da Istanbul. L'aspetto rilevante delle innovazioni ideologiche di Abdülhamid II, dunque, è il coinvolgimento delle masse nella sfera pubblica, operato grazie alla stampa come mezzo di diffusione delle idee. Accanto alla stampa, nondimeno, si trovano la propaganda, fatta di dichiarazioni, decorazioni e cerimonie; la scuola; e l'esercito. Scuola ed esercito, infatti, al pari della stampa, non hanno solo il compito di educare le nuove generazioni ma hanno anche il compito di consolidare l'identità ottomana e il sentimento patriottico di appartenenza all'Impero. L'educazione, fin dall'inizio delle Tanzimat; è sempre stata parte della mission civilisatrice degli uomini di Stato ottomani ma durante l'era Hamidiana diventa un fenomeno di massa ${ }^{15}$ rendendo l'Impero un «educator state» con un programma sistematico di educazione dei soggetti che intende trasformare in cittadini ${ }^{16}$.

Tra il 1880 e il 1885, pertanto, emerge un nuovo Stato e una nuova società i cui nessi con il passato ottomano-Islamico si fanno più forti, sebbene Abdülhamid II vi attribuisca dei caratteri peculiari ${ }^{17}$, che si palesano come un esempio di invenzione della

${ }^{11}$ Sui limiti delle Tanzimat vedi ABU-MANNEH, Butrus, «The Sultan and the Bureaucracy: The Anti-Tanzimat Concepts of Grand Vizier Mahmud Nedim Pasa», in International Journal of Middle East Studies, 22, 3/1990, pp. 257-274.

${ }^{12}$ Sugli ayan vedi MUGE GOÇEK, Fatma, Rise of the Bourgeoisie, Demise of Empire. Ottoman Westernization and Social Change, Oxford-New York, Oxford University Press, 1996; KARPAT, Kemal H., op.cit., pp. 92-93.

${ }_{13}$ La commistione tra modernità e Islam viene inaugurato dai Giovani Ottomani attraverso l'idea di Meşveret. MARDIN, Serif, The Genesis of Young Ottoman Thought: a Study in the Modernization of Turkish Political Ideas, Princeton, Princeton University press, 1962.

${ }^{14}$ GELVIN, James L., Storia del Medio Oriente Moderno, Torino, Einaudi, 2009, pp. 94-113.

15 DERINGIL, Selim, op.cit., p. 94.

${ }^{16}$ Ibidem, p. 93.

17 GEORGEON, François, «Le Sultan cache, réclusion du Sultan et mise en scène du pouvoir à l'époque d'Abdülhamid II (1876-1909)», in Turcica, 29, 1997, pp. 93-124; GEORGEON, François, Les Usage politique du Ramadan, de l'Empire Ottoman à la République de Turquie, 
tradizione. La particolarità patriottica dell'esperienza ottomano-turca sta proprio in questa situazione magmatica, in cui tutte le vie sono percorribili e vengono rielaborate in un discorso del tutto autonomo il cui scopo principale è la salvezza dell'Impero Ottomano. Variabile decisiva, in relazione alle contingenze storiche, è il peso della componente turca e musulmana all'interno dell'Impero divenuta dominante rispetto alle componenti cristiane che si trasformano in vere e proprie minoranze. Un cortocircuito che causa una costante evoluzione del discorso patriottico il quale assume forme di volta in volta differenti. Per comprendere appieno l'età Hamidiana, quindi, la si deve considerare come un luogo di fermento ideologico in cui, da un lato, culmina l'esperienza ottomanista delle Tanzimat (1876-78); dall'altro, si sviluppa la corrente Islamista o Panislamista nata da una costola dell'Ottomanismo (1880-90) ${ }^{18}$; e, infine, emerge un chiaro discorso patriottico al centro della quale si pone il tentativo del Sultano e delle élite di immaginare una nuova identità rispondente alle nuove necessità sociopolitiche che la modernità ha posto di fronte all'Impero.

\section{Abdülhamid II e il simbolismo ottomano}

Abdülhamid «credeva onestamente che le istituzioni democratiche introdotte con la Costituzione del 1876 avrebbero aiutato il suo sforzo di portare a compimento le Tanzimat»19. Questa sua linea democratica, tuttavia, viene subito vinta dalla lenta ed irregolare dinamica costituzionale e parlamentare, nonché dalla difficile situazione internazionale ${ }^{20}$; fattori che «lo convinsero, che soltanto attraverso un governo autonomo si poteva ottenere il rinnovamento dell'Impero» ${ }^{21}$. Una tesi avvalorata dal desiderio di ridare vigore al "grande malato", rendendolo uno Stato forte, come il Sultano ricorda in Pensées et souvenirs:

plus nous nous replierons sur nous-mêmes, plus nous nous concentrerons et plus vite notre faiblesse et notre "maladie" seraient surmontées. L'Europe verra alors

in GEORGEON, François, Sous le Signe des Réforme, État et Société de l'Empire Ottoman à la Turquie kémaliste (1789-1939), Istanbul, ISIS, 2009; KARPAT, Kemal H., op. cit.

${ }_{18}$ HANIOĞLU, M. Sukru, Turkism and the Young Turks 1889-1908, in KIESER, Hans-Lukas (eds), Turkey beyond Nationalism, towards Post-Nationalist Identities, London, I. B. Tauris, 2006, p. 7.

19 BOMBACI, Alessandro, SHAW, Stanfor J., L'Impero Ottomano, in BOMBACI, Alessandro, SHAW, Stanfor J. (a cura di), Nuova Storia Universale dei popoli e delle civiltà, vol. VI, t. II, Torino, UTET, 1981, p. 537.

${ }^{20}$ Il regno di Abdülhamid, di fatto, inizia nel pieno della crisi Balcanica del 1875-1878, e farà i suoi primi passi durante la guerra Russo-Turca del 1877-78 e il successivo Trattato di Berlino del 1878.

${ }^{21}$ BOMBACI, Alessandro, SHAW, Stanfor J., op. cit., p. 537. 
que "l'Homme malade" dont on s'est tant moqué deviendra un "Homme fort", quand il aura puisé de nouvelles force à l'intérieur ${ }^{22}$.

Questa svolta autoritaria riprende la via intrapresa da Selim III (1789-1807) e da Mahmud II (1808-1839), riaffermando la preminenza del Sultano sulla Sublime Porta $^{23}$, come ricorda lo stesso Abdülhamid:

Ho fatto un errore quando ho voluto imitare mio padre, Abdülmecit, che tentò di riformare [l'Impero] attraverso la persuasione e le istituzioni liberali. Avrei dovuto seguire le orme di mio nonno, il Sultano Mahamut. Come lui ho capito che è solo con la forza che uno può governare il popolo che Dio gli affidato ${ }^{24}$.

L'autoritarismo, tuttavia, non è negativo25. Come ricorda Paul Baudin (1896) elogiando il Sultano per «gli immensi benefici» ottenuti dall'Impero Ottomano, Abdülhamid II implementa numerose riforme che i burocrati delle Tanzimat non sono stati in grado di attuare:

le gouvernement, l'administration, les finances, la guerre, la marine, la justice, l'instruction publique, les travaux publics, le commerce et l'industrie, toutes ces différentes branches du mécanisme de l'État fourniront autant de preuves éclatants du progrès extraordinaire que, grâce à l'initiative féconde du souverain, l'Empire a faites en prospérité matérielle, intellectuelle et morale ${ }^{26}$.

Il sistema Hamidiano, dunque, riposa su una contraddizione fondamentale. Da un lato Abdülhamid cerca di instaurare (o restaurare) un potere basato su un'autorità di tipo "tradizionale", ma dall'altro, continuando a modernizzare l'amministrazione, e

${ }_{22}$ ALI VAHBI, Bey, Avant la Débâcle de la Turquie. Pensées et Souvenirs de l'ex Sultan Abdülhamid, Paris, Attinger, 1916 cit. in GEORGEON, François, Abdülhamid II, cit. p. 263. Nelle parole di Abdülhamid II si può intravedere una propensione ad alcuni temi patriottici, a cominciare dalla salvezza dell'Impero. Cfr. anche gli articoli di PARNASSIAN, Boghos, «Bulgares et Grecs», in Osmanli, 4 gennaio 1883; SADIK, «Le XIVe Siècle de l'ère de l'Hégire», in Osmanli, 16 novembre 1882, i quali si concentrano sul desiderio che l'Impero Ottomano fosse lasciato tranquillo a lavorare per la sua riforma e rigenerazione.

${ }^{23}$ Lo scontro tra questi due poli di potere si può rilevare durante tutto l'arco della storia ottomana. Quello che maggiormente colpisce è la rapidità del cambiamento. In poco meno di un secolo, assistiamo all'accentramento di Mahmud II, al dominio della Sublime Porta durante le Tanzimat, per poi ritornare ad un netto assolutismo, seppur "assolutismo costituzionale".

24 ABDÜLHAMID II, Zabit ceridesi, vol. 2, p. 401, citato in SHAW, Stanford J., SHAW, Ezel Kural, History of the Ottoman Empire and Modern Turkey, Cambridge-London-New YorkMelbourne, Cambridge University press, 1977, p. 213.

25 Sulle influenze delle Tanzimat e delle idee Europee sulla personalità di Abdülhamid, cfr. GEORGEON, François, Abdülhamid II, cit., pp. 19-40.

${ }^{26}$ BAUDIN, Paul, op. cit., p. 116. 
l'insegnamento contribuisce a rafforzare l'autorità della burocrazia ${ }^{27}$. Un autorità controbilanciata dalla vera novità del regno Hamidiano: l'adozione dell'Islam come simbolo politico identitario e patriottico. Quello che in apparenza sembra un passo indietro rispetto alle Tanzimat nella strada verso la democrazia liberale, quindi, è stato un deciso passo avanti verso l'evoluzione sociopolitica del mondo turco-ottomano e islamico.

La figura di Abdülhamid, tuttavia, si dimostra ben diversa rispetto ai Sultani che lo hanno preceduto. Sebbene l'apparenza tradisca un certo tradizionalismo nei modi e nei pensieri28, Abdulhamid passava molto tempo fuori dai suoi appartamenti di Dolmabahçe e sfruttava questa opportunità per frequentare Ottomani di ogni classe sociale e persino alcuni stranieri, discutendo con loro i problemi dell'Impero e come potevano essere risolti29. A differenza dei suoi predecessori il Sultano adotta i benefici dati dalla modernità; nei suoi primi anni di regno (1876-1885), si mescola frequentemente con i suoi sudditi, al punto che i cittadini di Costantinopoli si abituarono a vederlo passeggiare a cavallo per la città o in Caicco diretto alle Acque Dolci d'Asia ${ }^{30}$. Un atteggiamento che gli permette di conoscere e comprendere, la situazione del popolo ottomano e i problemi a cui deve porre rimedio. Accortezza che gli consente di capire come i suoi sudditi considerino il loro orgoglio e la loro dignità nazionale più rilevanti della proprietà o della vita ${ }^{31}$.

Il comportamento di Abdülhamid II e il suo ritiro dalla vita pubblica in un tempo relativamente breve, tuttavia, sono un ottimo indicatore della ristrutturazione ideologica e politica che si sta sviluppando nell'Impero Ottomano. Il processo di allontanamento del Sultano dal popolo creò una contraddizione al cuore della stessa concezione del potere statale di Abdülhamid II: da un lato, il regime Hamidiano tenta in ogni modo di penetrare in profondità nella vita quotidiana della società; d'altro lato, l'ossessione di Abdülhamid per la sua sicurezza ha limitato le sue epifanie ${ }^{2}$. A partire dal 1880, nondimeno, emerge la presenza di un nesso sempre più stretto tra la "sparizione" del Sultano dallo spazio pubblico e la deriva del suo regime autoritario 33. Abdülhamid II, riprendendo la consuetudine dei Sultani ottomani di ritirarsi dalla vita

\footnotetext{
27 GEORGEON, François, Le Sultan cache, in Sous le Signe des Réforme, cit., pp. 41-42.

28 «During his years as prince, he seems to have distinguished himself among his brothers by avoiding the style European frivolity that was entering the palace under his father's influence, avoiding the extravagance to the point of parsimony». In SHAW, Stanford J., SHAW, Ezel Kural, History of the Ottoman Empire and Modern Turkey, cit., p. 172.

29 Ibidem.

$3^{\circ}$ HASLIP, Joan, The Sultan, the Life of Abdülhamid, cit., p. 137.

${ }^{31}$ Ibidem, p. 99.

${ }^{2}$ DERINGIL, Selim, op. cit., p. 18.

33 GEORGEON, François, Le Sultan cache, in Sous le Signe des Réforme, cit., p. 35.
} 
pubblica, sfrutterà la sua fobia diventando, agli occhi del suo popolo, una figura mitica34. Contingenza che sviluppa la simbologia del potere con una ricaduta politica significativa: meno il Sultano era visibile e più si dimostrava necessario che il potere affermasse la sua presenza35. Sebbene l'atto di ritirarsi dalla scena pubblica trovi le sue origini già nel XVII secolo, quando i Sultani abbandonarono il loro ruolo di condottieri trasformandosi in icone cerimoniali36, con Abdülhamid II assume dei chiari significati patriottici. Per questo, la comunicazione con il popolo e con il mondo esterno si sviluppa attraverso un sistema di simboli basati su motivi Islamici da cui si ricava una forte risonanza emotiva in grado di coinvolgere le masse 37 : le epifanie del Selamlik e le ricorrenze religiose. Questo sistema di apparizioni e festività tradizionali viene ripensato in chiave patriottica, rendendo il Sultano un simbolo dell'autorità statale ${ }^{38}$. Un esempio di questa nuova simbologia ci viene fornito dal giornale «Stamboul», il quale descrive in più occasioni l'epifania di Abdülhamid II durante il Selamlik39, e si sofferma sulle cerimonie per la circoncisione dei principi imperiali $4^{\circ}$ e sulle celebrazioni delle principali festività Musulmane.

Accanto alle celebrazioni "tradizionali" 41 , dal 1880, emergono, con un'importanza simbolica fondamentale, anche i festeggiamenti per il compleanno e l'anniversario dell'ascesa al trono del Sultano istituiti da Abdülhamid II ${ }^{42}$ al fine di creare una nuova

\footnotetext{
34 DERINGIL, Selim, op. cit., p. 18.

35 GEORGEON, François, "Le Sultan cache”, in ID., Sous le Signe des Réforme, cit., p. 35.

${ }^{6} 6$ GELVIN, James L., op. cit., p. 41.

37 DERINGIL, Selim, op. cit., p. 18.

${ }^{38}$ HASLIP, Joan, The Sultan, the Life of Abdülhamid, cit., p. 85. A proposito della ripresa delle "tradizioni" islamiche e del simbolismo religioso legato alla guida della comunità Musulmana cfr. anche: CRONE, Patricia; HINDS, Martin, God's Caliph, Religious Authority in the first century of Islam, Cambridge, Cambridge University Press, 1986; ROSENTHAL, Erwin I. J., Political Thought in Medieval Islam, Cambridge, Cambridge University Press, 1968.

39 Lo «Stamboul» pubblicherà diversi articoli sulla ricorrenza del Selamlik, rilevanti in quanto espressione del valore simbolico attribuito all'epifania del Sultano dal popolo della capitale ottomana. Cfr.: «Le Sultan» del 25 marzo 1882 e «Le Selamlik» del 29 aprile 1882, in cui si può trovare una descrizione abbastanza dettagliata della cerimonia e anche «Le Sultan», Stamboul, 20 giugno 1881, p. 1.

$4^{\circ}$ Cfr. la serie di articoli e trafiletti «Circoncision des princes impériaux», in Stamboul, 3 aprile 1882; 23 e 31 ottobre; 9 novembre e 7 dicembre 1883. Gli articoli propongono un dato simbolicamente significativo: l'elogio della parsimonia di Abdülhamid II, considerata lo specchio della sua oculata amministrazione economica, segno di cambiamento e riforma.

${ }^{41}$ Cfr. "Hirkai-Cherif», Osmanli, 3 agosto 1882, articolo anonimo in cui viene descritta la visita di Abdülhamid al mantello del Profeta conservato nel palazzo del Topkapi; e la serie di articoli dello Stamboul dal titolo «Le Ramazan», in cui si descrivono i festeggiamenti di questa ricorrenza musulmana come nel pezzo del 27 luglio 1881.

42 «Le Sultan», in Stamboul, 14 e 15 luglio 1881. Articoli per i festeggiamenti del $41^{\circ}$ compleanno del Sultano, le celebrazioni per la venerazione del vessillo imperiale da consegnare ai pellegrini diretti alla Mecca e i progetti di ristrutturazione del mausoleo meccano. A questi articoli segue la descrizione dei festeggiamenti per l'anniversario della salita al trono di Abdülhamid II, «Le Sultan», in Stamboul, 1 settembre 1881. L'importanza di questo nuovo simbolismo civile è riscontrabile anche nelle pagine della rivista «L'Orient», edita negli anni 1890: L'Orient,
} 
simbologia civile in grado di infondere a tutti i cittadini un sentimento di unità e identità. L’importanza di queste ricorrenze è testimoniata da un altro giornale l'«Osmanli», il quale in due editoriali43 celebra il Sultano insistendo sul nesso simbolico e metaforico tra Abdülhamid II e l'Impero Ottomano, per cui il Sultano non è solo il padre degli Ottomani, ma anche personificazione dell'Impero: «Le peuple Ottoman tout entier partage la joie et les vœux du Souverain, et désire ardentement que le ciel couronne du succès les efforts de sa Majesté pour le bonheur et la prospérité de l'Empire»44.

Queste celebrazioni dimostrano che Abdülhamid II conosce e sfrutta le tematiche simboliche e patriottiche vigenti in Europa, come la sacralizzazione della persona del re e la "personificazione della Nazione". Il simbolismo legato alla figura del Sultano - sia esso tradizionale, moderno o creato ex-post - si dimostra, quindi, un fattore estremamente rilevante della vita e della politica di Abdülhamid II, contribuendo a creare un immaginario patriottico per rappresentare le nuove dinamiche sociopolitiche dell'Impero. Ciò che si evince dalla stampa francofona - «Stamboul» e «Osmanli» - è lo stretto legame e la rapida evoluzione, tra il 1878 e il 1885, di un sentimento e di un simbolismo patriottici con tratti locali che sviluppano un discorso patriottico assimilabile a quello Europeo. In questo processo di formazione di un’identità immaginata il ruolo della stampa francofona è fondamentale, non solo perché traduce la simbologia Hamidiana, ma perché diffonde i nuovi simboli in ogni angolo dell'Impero e in Europa ${ }^{45}$. La diffusione di questi simboli permette alle élites imperiali di rappresentarsi in un modo del tutto nuovo, mettendo in luce quelle forze sociali e politiche interne all'Impero in grado di colmare il divario con l'Europa. L'invenzione di questa nuova tradizione non è fatta solo di feste e ricorrenze, è fatta anche di simboli tangibili, come dimostra l'utilizzo di alcuni emblemi nazionali quali: la bandiera

supplemento al 31 agosto 1890, p. 2-4; il numero del 4/16 marzo 1892; oppure il numero del 31 agosto 1894 .

43 KIAMIL, Abdullah, «L'Anniversaire de la naissance de S. M. l'Empereur», in Osmanli, 21 luglio 1881; MIDHAT, Ahmet, «Anniversaire de l'avènement au trône de sa Majesté l'Empereur et Khalife», in Osmanli, 7 settembre 1882.

${ }_{44}$ KIAMIL, Abdullah, "L'Anniversaire de la naissance de S. M. l'Empereur», in Osmanli, 21 luglio 1881. Sulla stessa linea cfr. l'elogio in SALIH, «Les Aspirations helléniques», in Osmanli, 7 marzo 1881: «Souverain magnanime des Ottomans [...] n'a cessé de donner à l'Europe et au monde entier des preuves évidente de ses intention généreuses et pacifiques, de son désir d’inaugurer une ère nouvelle». [corsivo mio, ndr.]

45 Il ruolo della stampa in lingua francese è duplice. Da un alto, il francese, in quanto koiné e lingua diplomatica dell'Impero, si propone come un medium privilegiato di diffusione delle idee Europee attraverso i giornali. Dall'altro, si dimostra uno strumento necessario allo sviluppo di una terminologia patriottica dal momento che il lessico francese dell'epoca era più ricco di termini e concetti patriottico-nazionali di quello ottomano-turco. Nondimeno, va ricordato che anche grazie a questo duplice ruolo della lingua e della stampa francofona il lessico patriottico si è diffuso e interiorizzato nella società e nella lingua ottomane. 
"nazionale", con stella e mezzaluna bianche su fondo rosso; lo stemma araldico ${ }^{46}$, in cui alla Tuğra del sultano si affiancano la bandiera rossa ottomana e il vessillo verde dell'Islam con stella e mezzaluna bianche47; l'inno imperiale e le marce militari dei giannizzeri riarrangiate e affiancate da nuove composizioni prodotte da musicisti moderni, come il fratello di Donizetti48; le medaglie e le onorificenze civili e militari ${ }^{49}$; senza dimenticare le immagini dell'Impero e dell'Sultano-Califfo50.

Conferma dell'adozione di questi simboli ci viene fornita ancora una volta dalla stampa francofona. Nelle pagine dell'«Osmanli» troviamo alcuni articoli sull'introduzione della bandiera ottomana nelle provincie: un breve trafiletto anonimo, in cui si esalta la rinnovata esposizione del vessillo ottomano alla Mecca51; e l'articolo di Sadik, Les Couleurs du Pavillon Rouméliote, del 25 settembre 1882, in cui si riportano le lamentele dei Musulmani della Rumelia Orientale a causa del divieto dell'esposizione della bandiera "nazionale" ottomana imposto dall'amministrazione bulgara. L'articolo è importante non tanto per la querelle provinciale, ma per la definizione in senso nazionale delle due bandiere e per la descrizione di quella ottomana: una «bandiera rossa con mezzaluna e stella bianche»52. Una descrizione riproposta anche dallo «Stamboul», il quale, in un articolo pubblicato il 10 febbraio 1885, riconosce la bandiera ottomana, definita come nazionale, quale simbolo della nuova identità imperiale voluta da Abdülhamid II e pertanto sottolinea la necessità di esporla in ogni porto e città dell'Impero53.

46 «One of the most notable symbols of the renewed emphasis on power and ceremonial in the late nineteenth century was heraldry. The Sublime State (devlet-i aliyye) was symbolized by the coat of arms of the House of Osman (arma-i Osmani). The design had been commissioned from an Italian artist by Mahmud II. By the time Abdülhamid II came to sit on the Ottoman throne, it was such a well-established part of the Ottoman official symbolism». In DERINGIL, Selim, op. cit., p. 26.

47 «Armoires Impériales», in Stamboul, 20 aprile 1882.

48 A proposito della musica europea e dei musicisti italiani al servizio dei Sultani ottomani si consultino gli interessanti articoli di ARACI, Emre, Da Donizetti a Guatelli. Musicisti italiani alla corte ottomana, in DE GASPERI, Attilio, FERRAZZA, Roberta, Italiani di Istanbul, Figure, comunità e istituzioni dalle Riforme alla Repubblica 1839-1923, Torino, Fondazione Giovanni Agnelli Edizioni, 2007, pp. 273-280; ÖZTÜRK, Nazende, Una famiglia di musicisti di Istanbul: i Lombardi, in DE GASPERI, Attilio, FERRAZZA, Roberta, op.cit., pp. 281-285.

49 Sono numerose le medaglie e le onorificenze distribuite negli anni Ottanta del XIX secolo, segno evidente della fidelizzazione al Sultano della nuova classe dirigente.

50 Si deve sottolineare l'importanza avuta dalla fotografia durante tutto il regno Hamidiano. Oltre ad essere una passione personale di Abdülhamid II, la fotografia riveste un ruolo simbolico durante gli anni 1880 e 1890 . La sua funzione è quella di consentire il controllo dell'Impero senza abbandonare Istanbul. La fotografia, tuttavia, veniva usata anche come strumento per rappresentare e diffondere all'estero le riforme e le novità introdotte nell'Impero. La fotografia, come la stampa, pertanto, svolge un ruolo fondamentale nella costruzione della nuova immagine e identità ottomane alla fine del XIX secolo.

51 «Un Nouveau succès éclatant du Halifax Ottoman», in Osmanli, 16 maggio 1881, p. 2.

$5^{2}$ SADIK, «Les Couleurs du Pavillon Rouméliote», in Osmanli, 25 settembre 1882, p. 2.

53 Stamboul, 10 febbraio 1885, p. 2. 
Il giornale perota non si ferma qui e, tra il 1881 e il 1885, propone ulteriori esempi di questa nuova simbologia patriottica. Innanzitutto attraverso la sua rubrica quotidiana Décorations, in cui riporta i nomi di tutti coloro che hanno beneficiato di premi e riconoscimenti imperiali. Tra questi spiccano: il trafiletto del 2 gennaio 1879, «La Nouvelle décoration», dove si elogia e descrive la nuova decorazione ottomana, istituita per coloro che «ont rendu de grands service à l'Etat, soit comme militaires, soit comme civils»; e il trafiletto del 27 settembre 1883 in cui si descrive l'adozione di una nuova decorazione. Ulteriore esempio della nuova simbologia civile viene fornito dallo «Stamboul» nell'articolo dell'8 marzo 1881, dove si descrivono le reazioni all'inno "nazionale" ottomano intonato durante la cerimonia in onore di Tevfik Paşa. Una segnalazione che testimonia ancora una volta come le élite e il governo imperiale stiano adottando tutto il bagaglio di simboli e strumenti del patriottismo vigente in Europa e li stiano adeguando alla realtà sociale imperiale.

Il simbolismo del potere Hamidiano si può suddividere in quattro categorie, tre delle quali sono strettamente connesse con la figura del Sultano e il suo Palazzo. Nella prima di queste categorie rientrano i simboli legati alla sacralità della persona del Sultano-Califfo come: lo stemma sugli edifici pubblici, la musica ufficiale, le cerimonie e i lavori pubblici. Tutte cose che riflettono in modo diretto la gloria e il potere dello Stato ottomano. In Secondo luogo, si trovano le manifestazioni della beneficenza imperiale: le decorazioni, i doni di copie del Corano e i cerimoniali di corte 54 . In terzo luogo c'è il simbolismo legato agli oggetti religiosi e artistici come le opere calligrafiche di grandi uomini Musulmani. Infine, la quarta categoria concerne il simbolismo della lingua nella documentazione ufficiale ottomana55. La questione della lingua consente di capire, attraverso frasi o parole chiave usate frequentemente nei documenti ufficiali e nella stampa, in che modo le élites Hamidiane concettualizzino il discorso patriottico del Sultano.

Il patriottismo di Abdülhamid II, tuttavia, non si limita agli aspetti sociali e immateriali ma, rivisitando il modello delle corti europee, si completa con un vasto progetto edile per Istanbul. Una trasformazione che non solo propone un nuovo panorama urbano nella capitale, ma simboleggia anche lo stretto legame che si sta

54 Relativamente ai cerimoniali di corte cfr. la serie di articoli «Etiquette consulaire», in Stamboul, 23 e 29 dicembre 1881; 8 febbraio 1882; 11 e 23 marzo 1882; 15 aprile 1882; e, infine, 15 e 29 settembre 1883. Relativamente a questo tema si veda anche la lettera firmata "un Turco", pubblicata dallo «Stamboul» il 24 marzo 1881 in cui viene smentita la notizia diffusa dal giornale italiano «Fanfulla», secondo cui gli ambasciatori Ottomani a Roma avrebbero partecipato ad un ricevimento ufficiale in frac, tipico abbigliamento occidentale, invece di indossare il costume nazionale (corsivo mio) composto da stambouline e fez.

55 DERINGIL, Selim, op.cit., p. 21. 
instaurando tra popolo e Sultano. L'aspetto di Istanbul si rimodella attraverso la costruzione di scuole, caserme, porti e han. Lo skyline della capitale si sviluppa con una nuova architettura urbana basata sulla costruzione di nuove case in pietra e muratura, e gli assi e gli equilibri urbani non gravitano più attorno al Topkapi e ai quartieri Musulmani di Fathi e Sultanahmet, ma bensì sui quartieri euro-levantini di Galata e Pera e sulle nuove periferie a nord di essi Nisantaşi, Beşiktaş e Şişli56.

\section{Il patriottismo Hamidiano difronte all'Europa}

«Le Sultan cache»57, pertanto, nonostante si mostri poco al suo popolo riesce attraverso un simbolismo e una propaganda sistematica a trasmettere un messaggio ideologico legato alla sua persona e alla sua immagine quale padre di tutti gli Ottomani e sovrano di tutto l'Impero, che lo rende, in absentia, il primo simbolo del patriottismo imperiale. Abdülhamid, infatti, «[for] the cheering crowds [...] was a symbol rather than a man»58; per i suoi sudditi, è il «buon padre» Hamid, colui che dona in beneficienza ingenti somme, vestiti e viveri alle vittime dei frequenti incendi che devastavano Istanbul59. Un'immagine simbolica che Abdülhamid cerca di sfruttare al fine di stringere a sé i suoi sudditi, nel tentativo estremo di creare un popolo e una società coesi dall'insieme di razze, lingue e religioni di un Impero cosmopolita ${ }^{60}$. Il regno Hamidiano, quindi, va inteso come un periodo di passaggio, in cui si pongono le basi per una nuova statualità, che permetterà all'Impero Ottomano di rinascere come Stato nazionale.

Essendo «the only European Muslim Power» ${ }^{61}$, l’immagine che l'Impero Ottomano vuole dare di sé risulta decisiva nel contesto socio-politico mediterraneo. Un tema a cui l'«Osmanli» dedica numerosi articoli, rivolti sia all'opinione pubblica interna, sia a quella Europea, con il fine di proporre un'immagine accattivante degli Ottomani e difendere l'Impero ${ }^{62}$.

${ }^{56}$ La retorica architettonica simbolo della nuova identità Ottomana si rileva in alcune opere dallo stile neo-ottomano - frutto del sincretismo tra la tradizione ottomana e la modernità dell'architetto Raimondo D'Aronco, attivo a cavallo tra XIX e XX secolo.

57 GEORGEON, François, Abdülhamid II, cit.

${ }^{8}$ HASLIP, Joan, The Sultan, the Life of Abdülhamid, cit., p. 205.

59 Ibidem, p. 243.

60 «The liquidity and fluidity of identities in those centuries is hard to imagine in the national age». KAFADAR, Cemal, Between Two Worlds, the Construction of the Ottoman State, Berkeley-Los Angeles-London, University of California press, 1995, p. 28.

${ }^{61}$ DERINGIL, Selim, op. cit., p. 1.

${ }^{62}$ SALIH, «L'Opinion de l'internonce Busbèq sur l'Empire Ottoman», in Osmanli, 26 maggio 1881, p. 2; SALIH, «Un Blue book sur le Kurdes», in Osmanli, 2 giugno 1881, pp. 1-2; SULEIMAN, «Bienfaisantes fondations», in Osmanli, 16 marzo 1882, p. 1; SALIH, «M. 
Oltre al dialogo con l'opinione pubblica l'«Osmanli» sviluppa anche altri temi volti a persuadere gli Europei del progresso ottomano e delle potenzialità della civilizzazione orientale. In tutti questi articoli resta evidente il fine patriottico del giornale che non solo difende lo Stato e il suo operato ma anche il suo sistema ideologico e culturale. Come sottolinea Salih, l'Impero: «ne désire que il progrès et la régénération de l'Orient par les efforts pacifiques des toutes les peuples que habitent ces régions. N'est nullement ennemie de l'Europe et de sa civilisation, dans ce qu'elle a de bon et d'utile»63. Anzi, secondo lo stesso Salih: «l'Europe a un intérêt moral de premier ordre à ce que l'Orient entre résolument dans la voie du progrès qu'il développe sa vie nationale et qu'il applique les mêmes principes qui régissent toutes les nations civilisées de nos jours» 64 .

Abdülhamid II, nel suo processo di rinnovamento e costruzione dello Stato, quindi, cerca di immaginare una nuova comunità e di inventare una nuova tradizione adatta ad essa e alle contingenze del suo Impero. Un progetto che si realizza attraverso la stampa quotidiana ${ }^{65}$, la quale sviluppa i mezzi tecnici per ripresentare il tipo di comunità immaginata rappresentato dalla Nazione ${ }^{66}$. Il giornale e la stampa, dunque, sono strumenti fondamentali nello sviluppo delle teorie patriottiche ottomane, come dimostrano l' «Osmanli» e lo «Stamboul», i quali vanno intesi come il principale luogo di comunicazione della società ottomana, in quanto "salotti" adatti alla conversazione e alla condivisione. Attraverso le pubblicazioni a stampa, pertanto, si può tracciare una linea di giunzione tra l'esperienza Europea e quella Ottomana, visto che l'Impero accede alla stampa quotidiana durante il XIX secolo, e la società tardottomana, come sostiene Koloğlu' ${ }^{67}$, è una società basata sulla cultura del giornale.

L'emergere di questo nuovo e peculiare discorso patriottico si può osservare in modo molto chiaro nelle pagine dell' «Osmanli», giornale turco-ottomano, in cui si trovano diversi articoli che giustificano atteggiamenti ed espressioni patriottici del governo, del popolo e della stampa ottomani:

Ashmead Bartlett sur la politique du cabinet anglais», in Osmanli, 31 agosto 1882, p. 2; SADIK, «Les Fonctionnaires allemands», in Osmanli, 2 ottobre 1882, p. 2.

${ }^{6}$ SALIH, «Un Nouveau droit des gens réaliste», in Osmanli, 16 febbraio 1882, p. 1.

${ }^{64} \mathrm{SALIH}$, «Les Intérêts de la civilisation en Egypte», in Osmanli, 27 luglio 1882, p. 1.

65 «Les Orientaux [...] communiquent entre eux beaucoup plus qu'on ne le croit généralement, ils emploient encore les procèdes anciens [...] Ils ont, en autre - et ceci est l'ouvre de la civilisation moderne et de l'esprit de progrès - des organes, des journaux imprimés». KIAMIL, Abdullah, «Le Régime arabe», in Osmanli, 30 maggio 1881, p. 2. [corsivo mio, ndr.]

66 ANDERSON, Benedict, Imagined Communities, Reflection on the Origin and Spread of Nationalism, London-New York, Verso, 1983, p. 25.

${ }^{67}$ KOLOGLU, Orhan, Le Premier journal officiel en français a Istanbul et ses répercussions en Europe, in CLAYER, Nathalie, POPOVIC, Alexandre, ZARCONE, Thierry, Presse turque et Presse de Turquie. Actes des colloques d'Istanbul, Istanbul-Paris, ISIS, 1992, pp. 3-13. 
personne ne peut douter que le patriotisme est le sentiment le plus élevé et le plus sacré qui puisse exister au monde. Mais, si le renouvellement et l'excitation d'un sentiment antique ne sert que à troubler l'état présent, le bonheur actuel, comme cet acte ne saurait être conforme aux principes conservateurs, tout gouvernement croit nécessaire de l'empêcher ${ }^{68}$.

L’importanza di questo passaggio non si trova solo nella constatazione dell'esistenza di un patriottismo ottomano, ma soprattutto nella difesa di questo patriottismo. Una difesa legittimata proprio dalle pratiche Europee e dalle modalità che gli Stati Europei adottano per difendersi contro i movimenti separatisti delle loro minoranze. Un concetto espresso anche nell'articolo «L'Expulsion des sujets hellènes du point de vue juridique»:

la Turquie a le même droit chez elle que tout autre gouvernement civilisé, et l'on ne pourrait soulever aucune exception contre elle. Dans l'intérêt public elle peut et elle doit prendre le même mesures de sécurité qui sont exercées par les autres gouvernements dans de circonstances certainement moins graves ${ }^{69}$.

Appare evidente che l'élite imperiale conosce bene le dinamiche patriottiche e rivendichi per sé e per il suo governo le medesime prerogative degli Stati Europei, i quali, con il loro approccio orientalista, non considerano uno Stato Musulmano al loro livello di civilizzazione. Le critiche Europee, tuttavia, non restano lettera morta e l'«Osmanli», risponde in questo modo:

chaque être organisé doit lutter pour son existence. C'est la loi de la nature, à la quelle n'échappent pas plus les sociétés humaines que les individus. [...] Un État, dans sa lutte pour l'existence, a besoin du concours éclairé et continuel de tous les citoyens $[. .$.$] c'est le travail national qui produit un ensemble de progrès dont les$ bienfaits rejaillissent sur le pays ${ }^{70}$.

Da questi esempi si può chiaramente osservare come le élites ottomane utilizzino dei concetti e una terminologia di tipo patriottico, i quali, adattati alla realtà imperiale,

68 MIDHAT, Ahmet, «Publications séditieuses», in Osmanli, 17 febbraio 1881, p. 1 [Corsivo mio].

69 SALIH, «L'Expulsion des sujets hellènes du point de vue juridique», in Osmanli, 3 marzo 1881, p. 1.

${ }^{70}$ SADIK, «La Lutte pour l'existence», in Osmanli, 7 dicembre 1882 p. 2. 
pongono le basi per quel discorso proto-nazionale turco emerso a partire dagli anni 1890 del XIX secolo. Pertanto, si può sostenere, appoggiandosi alle prove documentarie dell'«Osmanli», che negli anni di Abdülhamid II, grazie alla stampa ottomanofrancofona, si sviluppa il terreno adatto per la nascita di un discorso di tipo patriotticonazionale in stretta relazione con i modelli Europei ${ }^{71}$. Il patriottismo ottomano, nondimeno, non è solo difensivo, ma ha anche l'obiettivo di presentare l'Impero come uno Stato moderno e civilizzato. Un compito che l'«Osmanli» svolge con una serie di articoli, pubblicati dal 23 marzo 1882 al 15 maggio 1882, dal titolo «Avenir de l'Empire Ottoman» in cui, accanto agli elogi propagandistici al Sultano e all'Impero, viene descritta la situazione ottomana nel 1882 dal punto di vista politico, sociale ed economico, elogiando gli sforzi già compiuti e dando consigli per il futuro, in modo da garantire «prospérité à l'intérieur, [et] prestige à l'extérieur». Due principi di condotta che «ont toujours régi les Etats civilisés et régissent dès nos jour les Etats modernes» e sono «la devise à adopter par l'Empire Ottoman»72. Secondo l'«Osmanli», infatti: «l'avenir de l'Empire Ottoman, avenir considérable, réside tout entier dans l'adoption positive et l'adoption rationnelle des progrès intellectuels et matériels des autres nations à la civilisation ottomane, et à son peuple»73.

La stampa quotidiana si rivela uno strumento decisivo nel contesto socio-politico ottomano degli anni 1880-85, come medium per le idee Europee e per lo sviluppo della società civile ottomana, ma anche per il ruolo formativo ed educativo dei cittadini imperiali che si confrontano con le nuove ideologie politiche e la nuova identità promossa dal Sultano. Negli anni 1880, infatti, il Sultano mette in atto quello che Thiesse chiama «le processus de formation identitaire», che consiste nel «déterminer le patrimoine de chaque nation et à en diffuser le culte»74. A questo processo fa da corollario una serie di accorgimenti sociali, politici e tecnologici, quali: l'elaborazione di un simbolismo imperiale, la proclamazione di una nuova legislazione, la costruzione di ferrovie e telegrafi, i censimenti ${ }^{75}$, il controllo territoriale attraverso le mappe, la fondazione di musei, etc. Riforme che rendono l'Impero Ottomano uno Stato moderno

\footnotetext{
${ }^{71}$ Per comprendere come le élites di Istanbul erano in contatto con le dinamiche nazionali Europee cfr. SALIH, «Germains et Slaves», in Osmanli del 6 aprile 1882, p. 2. Qui si descrive il nazionalismo etnico tedesco, l'evolversi del Pan-Slavismo dopo il 1878, e anche i motivi principali della frizione tra le due ideologie e tra Russia e Germania, sostenendo che proprio a causa dei Balcani e della Bosnia scoppierà una guerra tra le due potenze.

72 Dr. Edwards, «Avenir de l'Empire Ottoman», Osmanli, 27 marzo 1882, p. 2.

73 Dr. Edwards, «Avenir de l'Empire Ottoman», Osmanli, 30 marzo 1882, p. 2.

74 THIESSE, Anne-Marie, La Création des identités nationales, Europe XVIII ${ }^{e}-X X^{e}$ siècle, Paris, Edition du Seuil, 1999'1, 2001, p. 12.

75 Riguardo al ruolo dei censimenti come mezzo amministrativo per migliorare i legami identitari: ANDERSON, Benedict, op. cit., pp. 163 et seq.
} 
e scuotono profondamente il modo in cui lo Stato immagina il suo dominio, la natura degli essere umani che governa, la geografia delle terre che domina e la legittimità dei suoi antenati ${ }^{7}$. La stampa aiuta a comprendere queste riforme e la loro diffusione come dimostra la serie di articoli sulle biblioteche "nazionali" di Istanbul dal titolo «Les Bibliothèques de Constantinople»77 e dagli articoli relativi al nuovo Museo Archeologico ${ }^{78}$ pubblicati dallo «Stamboul», in quanto simboli del regno Hamidiano e della nuova identità ottomana, a cui si sommano i due articoli pubblicati dall'«Osmanli» sugli archivi Ottomani79.

\section{Conclusioni}

Negli anni 1880-1885, il regno Hamidiano è teatro di un fermento politico e culturale, di cui la stampa è uno degli attori principali, e in cui muovono i primi passi alcune teorie e tematiche patriottiche e protonazionali che troveranno ampia diffusione nelle mentalità ottomano-turche all'inizio del XX secolo. Gli esempi della stampa francofona dimostrano l'esistenza e la coscienza da parte di tutti gli attori sociali di un discorso patriottico ben radicato all'interno della mentalità e della società Hamidiane. Tuttavia, nonostante l'utilizzo di termini quali Patria, Nazione, Patriottismo e via discorrendo, gli articoli della stampa francofona sottolineano più volte come non sia ancora possibile parlare di Nazionalismo strictu sensu. Gli obiettivi del Sultano e delle élites ottomane, infatti, sono dissimili da quelli nazionali, anche se nella retorica costruita e sviluppata da Abdülhamid II sono presenti molti dei temi e dei simboli che hanno guidato gli Europei nel loro cammino verso lo Stato-Nazione. È possibile affermare che il periodo intercorso tra il 1880 e il 1885 sia un periodo di incubazione per diverse teorie politiche - di differente origine, endogene ed esogene, conservatrici e

\footnotetext{
${ }^{76}$ ANDERSON, Benedict, op. cit., p. 164. A proposito del ruolo educativo-propagandistico delle mappe si veda anche il saggio di FORTNA, Benjamin C., Imperial Classroom, Islam, the State, and Education in the Late Ottoman Empire, Oxford-New York, Oxford University press, 2002, pp. 165-201. «It is no accident that the state emphasized the cartographic unity of its territory as it sought to promote a renewed sense of loyalty to the empire and its ruler». Ibidem, p. 166.

77 «Les Bibliothèques de Constantinople», in Stamboul, 15 marzo 188, p. 3; 13 maggio 1882, p. 3; 13 giugno 1882, p. 2; 10 luglio, p. 2; 24 agosto 1882, p. 3; «Bibliothèque Nationale», in Stamboul, 14 novembre 1882, p. 3; 29 dicembre 1883, p. 2; «Bibliothèques de la capitale», in Stamboul, 18 gennaio 1883, p. 3; «Bibliothèque publique», in Stamboul, 20 marzo 1884, p. 3; 12 aprile 1884, p. 3; 11 giugno 1884, p. 3. Cfr. HITZEL, Fréderic, «Manuscrits, Livres et Culture livresque à Istanbul», in HITZEL, Frederic (eds), Livre et Lecture dans le monde ottoman, in Revue des Mondes Musulman et de la Méditerranée, 87-88, 1999, pp. 19-38.

78 «Le Musée d'antiquités de Stamboul», in Stamboul 29 novembre 1883, p. 3; 10 e 13 dicembre 1883, p. 3 .

79 «Archives Turques», in Osmanli, 28 agosto 1882, p. 2; SADIK, «Les Archives», in Osmanli, 31 agosto 1882, p. 2; e «Les Archives des tribunaux turcs», in Stamboul, 25 agosto 1882, p. 2.
} 
moderniste - utilizzate in una forma del tutto innovativa rispondente a determinate contingenze storiche, sociali e politiche, di cui il patriottismo è solo una tra le tante.

Dalla stampa francofona emerge senza alcun dubbio la presenza di un discorso di tipo patriottico ma, al tempo stesso, è evidente come non si possa tracciare una linea di dipendenza diretta tra questo discorso e il successivo nazionalismo turco-kemalista. Quello che è indubbio sono le permanenze e la continuità con il periodo Hamidiano riscontrabili nella teoria politica sviluppata dai Giovani Turchi e da Mustafa Kemal. Sia i Giovani Turchi, sia Mustafa Kemal e gli intellettuali della prima Repubblica, infatti, sono nati, sono cresciuti ed hanno studiato nella società e nelle scuole Hamidiane ricevendone un’influenza ben definita, che si è poi sviluppata nel loro pensiero e nelle loro teorie politiche. Va comunque ricordato che i due sistemi sociali e politici sono motivati da contingenze storiche differenti. Pertanto, sebbene sia necessario analizzare e comprendere a fondo il regno di Abdülhamid II e soprattutto gli anni 1880-1885 per poter affrontare lo studio della prima fase repubblicana, non bisogna dimenticare che queste due fasi rappresentano due aspetti molto differenti della storia ottomano-turca. 


\section{* L'autore}

Luca Zuccolo è dottore in Storia Contemporanea presso il SUM (Istituto Italiano di Scienze Umane) di Napoli dove ha sviluppato una ricerca sulla stampa francofona ottomana e la sua rappresentazione dell'Impero d'Oriente. Già dottore magistrale in Storia d'Europa (Bologna, 2008), il suo campo di ricerca si rivolge allo sviluppo della modernità durante l'ultimo secolo dell'Impero Ottomano, al confronto/scontro tra modernità e tradizione in un contesto cosmopolita e allo sviluppo dei movimenti sociali che hanno preparato l'avvento della società turca contemporanea. Luca Zuccolo è il referente di Diacronie per la storia turca e ottomana.

URL: < http://www.studistorici.com/2010/12/o1/luca-zuccolo/ >

\section{Per citare questo articolo:}

ZUCCOLO, Luca, «ll patriottismo e la stampa ottomana», Diacronie. Studi di Storia Contemporanea : Le monarchie nell'età dei nazionalismi, 29/12/2013,

URL: < http://www.studistorici.com/2013/12/29/zuccolo_numero_16/ >

Diacronie Studi di Storia Contemporanea of www.diacronie.it

Risorsa digitale indipendente a carattere storiografico. Uscita trimestrale.

redazione.diacronie@hotmail.it

Comitato di redazione: Marco Abram - Jacopo Bassi - Luca Bufarale - Alessandro Cattunar - Elisa Grandi - Deborah Paci - Fausto Pietrancosta - Matteo Tomasoni - Luca Zuccolo

Diritti: gli articoli di Diacronie. Studi di Storia Contemporanea sono pubblicati sotto licenza Creative Commons 2.5.

Possono essere riprodotti a patto di non modificarne i contenuti e di non usarli per fini commerciali. La citazione di

estratti è comunque sempre autorizzata, nei limiti previsti dalla legge. 